\title{
Negative-Frequency Resonant Radiation
}

\author{
E. Rubino, ${ }^{1}$ J. McLenaghan, ${ }^{2}$ S. C. Kehr, ${ }^{2}$ F. Belgiorno, ${ }^{3}$ D. Townsend, ${ }^{4}$ S. Rohr, ${ }^{2}$ C. E. Kuklewicz, ${ }^{4}$ U. Leonhardt, ${ }^{2}$ \\ F. König, ${ }^{2, \dagger}$ and D. Faccio ${ }^{4, *}$ \\ ${ }^{1}$ Dipartimento di Scienza e Alta Tecnologia, Università dell'Insubria, Via Valleggio 11, IT-22100 Como, Italy \\ ${ }^{2}$ School of Physics and Astronomy, SUPA, University of St Andrews, North Haugh, St Andrews, KY16 9SS, United Kingdom \\ ${ }^{3}$ Dipartimento di Matematica, Politecnico di Milano, Piazza Leonardo 32,20133 Milano, Italy \\ ${ }^{4}$ School of Engineering and Physical Sciences, SUPA, Heriot-Watt University, Edinburgh EH14 4AS, United Kingdom
}

(Received 16 December 2011; published 18 June 2012)

\begin{abstract}
Optical solitons or solitonlike states shed light to blueshifted frequencies through a resonant emission process. We predict a mechanism by which a second propagating mode is generated. This mode, called negative resonant radiation, originates from the coupling of the soliton mode to the negative-frequency branch of the dispersion relation. Measurements in both bulk media and photonic-crystal fibers confirm our predictions.
\end{abstract}

DOI: 10.1103/PhysRevLett.108.253901

PACS numbers: 42.65.Ky, 05.45.Yv, 42.65.Hw

Introduction.-Resonant radiation (RR), often also referred to as dispersive-wave or Cherenkov radiation, is a nonlinear optical process by which a soliton propagating in an optical fiber in the presence of higher-order dispersion sheds light through a resonantlike process to a shifted frequency [1-5]. This process and the precise frequency of the RR is determined by a wave vector-matching condition between the dispersive resonant radiation and the soliton, i.e.,

$$
k\left(\omega_{\mathrm{RR}}\right)=k\left(\omega_{\mathrm{IN}}\right)+\left(\omega_{\mathrm{RR}}-\omega_{\mathrm{IN}}\right) / v+K_{\mathrm{NL}}\left(\omega_{\mathrm{IN}}\right),
$$

where $k=\omega n(\omega) / c$ is the wave vector at frequency $\omega$, $\omega_{\mathrm{IN}}$ and $\omega_{\mathrm{RR}}$ are the soliton (or input) and RR frequencies, $v$ is the soliton velocity, and $K_{\mathrm{NL}}=\omega_{\mathrm{IN}} n_{2} I / c$ is a nonlinear correction term ( $n_{2}$ is the nonlinear Kerr coefficient) that may be small or even negligible at low intensities, $I$ [5]. A very similar process occurs also in bulk media. The stationary 1D fiber soliton is now replaced by the stationary three-dimensional $X$ wave [6]. $X$ waves may form spontaneously in Kerr media at high enough powers in much the same way that solitons form spontaneously in a fiber [7,8]. A blueshifted peak will also be observed that will form one of the two $X$ wave tails: the whole $X$ wave, including the RR, is therefore described by Eq. (1) [9], which indeed reflects the nondispersive nature of the wave packet considered, i.e., the soliton in one dimension and the $X$ wave in three dimensions. A simple interpretation of Eq. (1) is derived by noting that RR generation in both one and three dimensions can be interpreted within a Bornapproximation scattering event, whereby the input pump pulse creates a scattering potential through the Kerr effect and then the pump light is self-scattered from this potential [10-12]. Equation (1) therefore reads as the momentum conservation relation that governs this scattering process.

Resonant-radiation frequency conversion describes an energy transfer between specific modes identified by Eq. (1) and the dispersion curve [4,5,9]. In 1D geometry, the soliton lies in the anomalous dispersion region and transfers energy to RR in the normal dispersion region, while in three dimensions it is possible to excite RR within the same dispersion region due to the intrinsic spatiotemporal dispersion of the $X$-wave states. However, to date only the positive frequency branch of the dispersion has been considered when it actually also has a branch at negative frequencies. This branch is usually neglected or even considered meaningless when in reality, as we show here, it may be of physical relevance and may host mode conversion to a new frequency. The fact that a mode on the negative branch of the dispersion relation may be excited has a number of important implications beyond the simple curiosity of the effect in itself. Indeed, light always oscillates with both positive and negative frequencies, but the negative-frequency part is directly related to its positive counterpart and seems redundant [13]. On the other hand, light particles (photons) have positive energies and are associated with positive frequencies only [14]. A process such as that highlighted here, which mixes positive and negative frequencies, will therefore change the number of photons, leading to amplification or even particle creation from the quantum vacuum $[15,16]$.

In this Letter, we show how, alongside the usual resonant-radiation spectral peak observed in many experiments, a second, further blueshifted peak is also predicted. This new peak may be explained as the result of the excitation of radiation that lies on the negative-frequency branch of the dispersion relation. We first explain why this radiation should be observed and then provide experimental evidence of what we call "negative-frequency resonant radiation" in both bulk media and photonic-crystal fibers.

Theory.-In order to show how the negative-frequency $\mathrm{RR}$ arises, we consider without any loss of generality a basic dispersion relation that contains higher-order terms such as in fused silica glass, shown in Fig. 1(a) (red curves). The dashed curves indicate the negative-frequency 
(a)
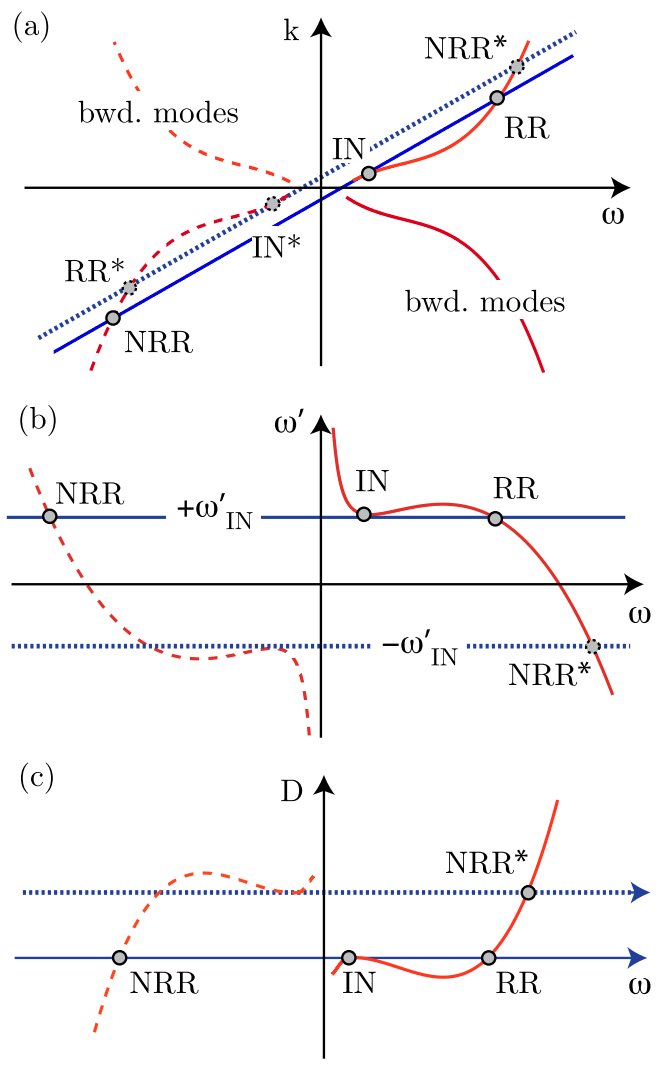

FIG. 1 (color online). Typical dispersion relation $k=k(\omega)$, e.g., for fused silica glass with second- and third-order dispersion, (a) in the laboratory reference frame and (b) in the reference frame comoving at the soliton velocity. (c) The dispersion $D=D(\omega)$, as described in the text. Dashed curves indicate the (laboratory frame) negative frequency branches of the dispersion relation. For simplicity, we take $K_{\mathrm{NL}}=0$.

branches. The phase-matching relation (1) (with $K_{\mathrm{NL}}=0$ ) can be solved graphically: the soliton term is a straight line (in blue in the figure) that intersects the dispersion relation in a number of points that define the allowed modes. We note that around $\omega=0$ both Eq. (1) and the dispersion relation should be expected to pass through $k(0)=0$. This is not of any concern for what follows as we always work at high frequencies, well within the region such that our equations describe the light propagation in the medium very precisely. Moreover, in the following we shall also neglect the top-left and bottom-right regions in the $(k, \omega)$ plot as these correspond to backward ("bwd") propagating modes that are not excited by the forward propagating input mode. The point indicated with IN is simply the input mode, or soliton mode. There is a second positivefrequency mode, $\mathrm{RR}$, that indicates the resonant-radiation mode. However, in the derivation of Eq. (1), no assumptions are made regarding the value of $\omega$, i.e., $\omega$ may run over both positive and negative values. We then see that there is also a negative-frequency mode (NRR) predicted by Eq. (1) yet always neglected. The object of this Letter is precisely this negative-frequency branch mode. All these various modes are easier to visualize in the comoving reference frame coordinates, as shown in Fig. 1(b). These curves are obtained from the original dispersion relation by transforming via a Doppler shift to the input pump or soliton comoving coordinate system $\omega^{\prime}=\gamma(\omega-v k)$, with $\gamma=1 / \sqrt{1-v^{2} / c^{2}}$, and $v=v_{g}$ is the soliton group velocity. Transforming also Eq. (1) to the comoving frame using the same relations gives $\omega^{\prime}=\omega_{\mathrm{IN}}^{\prime}$. In other words, momentum conservation in the laboratory reference frame corresponds to constant energy (in the sense that all modes have the same frequency) in the comoving reference frame. The allowed modes are therefore now found by simply tracing a horizontal line through the input soliton mode (that by definition, has zero group velocity in the comoving frame and thus lies at a local minimum) and, as before, looking for the intersections with the dispersion relation. The main point here is that the dispersion curves tell us that it should be possible, starting from two positive modes, IN and RR, to excite a third negative mode. Finally, in Fig. 1(c) we present the same relations in a format that has been used when describing optical solitons (e.g., Ref. [17]). By Taylor expanding the output wave vector $k\left(\omega_{\mathrm{RR}}\right)$ around $\omega_{\mathrm{IN}}$, the first two lowest-order terms cancel out in Eq. (1), which therefore reduces to $D(\omega)-K_{\mathrm{NL}}=0$, where the dispersion $D(\omega)=\sum\left(k_{n} / n !\right)$, with $n \geq 2$ and $k_{n}$ as the dispersion coefficients associated with the Taylorseries expansion [4,5]. (In Fig. 1, for simplicity, we are assuming $K_{\mathrm{NL}} \sim 0$.) In this case, we see that by including the negative-dispersion branch, as in the preceding figures, an additional intersection with the $\omega$ axis denotes the existence of the NRR mode.

When trying to assign a physical meaning to the negativefrequency mode, we should recall that in reality any electromagnetic field is a real-valued quantity that can be written as a sum of a complex term with its complex conjugate (both propagating in the same forward direction): $\quad E \sim \cos \omega t \propto \exp [+i(k z-\omega t)]+\exp [-i(k z-$ $\omega t)]$. However, considering only the modes obtained from the intersections with Eq. (1) (or equivalently, with $\omega^{\prime}=$ $+\omega_{\text {IN }}^{\prime}$ in the comoving frame) amounts to considering only the first complex term and neglecting the complex conjugate. In order to recover the full field, we obviously also need to sum the modes obtained from the intersections with the complex conjugate of Eq. (1) (or equivalently, $\omega^{\prime}=$ $\left.-\omega_{\text {IN }}^{\prime}\right)$ : these curves physically represent the momentum conservation condition, Eq. (1), for a scattering potential created by the complex-conjugate input pulse (IN*) and are shown as dashed lines in Fig. 1. The sum of NRR and NRR* in Fig. 1, therefore, will give a real-valued field with a positive frequency in the laboratory reference frame. Nevertheless, as explained above, the origin of this mode lies in the coupling of one or more modes on the positivefrequency branch of the dispersion relation to a mode that lies on the negative-frequency branch. We also note that the negative mode has a truly distinct frequency from all the other modes in Fig. 1 and, if it is generated, it should appear as a clearly distinct peak in the spectrum with a higher 
frequency than the RR mode. In analogy with the usual positive-frequency RR, we call this new mode "negativefrequency resonant radiation" (NRR).

We note that the fact that the negative RR mode is a solution to Eq. (1) does not, alone, imply that it will actually be excited. Similarly to the RR mode, the negative mode will only be excited if a sufficiently steep shock front is formed within the pump pulse. Indeed, this condition guarantees that the pulse contains spectral components at sufficiently blueshifted frequencies to actually seed both $R R$ and NRR generation. The main difference with respect to the RR mode is that the NRR mode is even further blueshifted, thus requiring even steeper shock fronts. This requirement may also be loosely understood in terms of the excitation of a mode that in the frequency domain is shifted far from the input mode. Therefore, in order for the IN mode energy to be scattered to the NRR mode, a sufficiently fast variation, i.e., a shock front, is required in the time domain. Indeed, preliminary numerical simulations, albeit in simplified setting (e.g., Ref. [18]), do indicate that the actual intensity of the negative mode depends critically on the steepness of the refractive index variation induced by the nonlinear Kerr effect.

Finally, we note that in the comoving frame both the RR mode and the NRR mode propagate with negative group velocities (as can be deduced from the slope of the dispersion curve at these frequencies), i.e., in the backward direction. The phase velocities of the two modes are however opposite to each other. Conversely, in the laboratory frame both the RR and the NRR modes have positive phase and group velocities, i.e., they both propagate in the forward direction (at slower group velocities with respect to the input mode).

Experiments.-We performed two sets of experiments in order to capture the formation of the negative RR mode: (i) in a bulk medium and (ii) in a few-millimeter-long photonic-crystal fiber. In the first experiment, we chose a 2-cm-long bulk calcium flouride sample $\left(\mathrm{CaF}_{2}\right)$ as host material. Light pulses of 60 -fs duration and $800-\mathrm{nm}$ carrier wavelength are provided by an amplified Ti:sapphire laser system of 1-kHz repetition rate. Under these conditions (3D geometry), we do not excite a soliton, but the nonlinearity will nevertheless excite a resonant instability that is governed by the same physics—and by Eq. (1) - as RR in optical fiber solitons [10-12]. We reshape the pulses into Bessel beams with a cone angle (in the medium) of $\theta=0.6^{\circ}$, using a conical lens of fused silica with $2^{\circ}$ base angle. The Bessel pulse in the sample moves with uniform speed $v=v_{g} / \cos \theta$, where $v_{g}$ denotes the group velocity of a Gaussian pulse of carrier wavelength $800 \mathrm{~nm}$. Moreover, the Bessel-beam geometry plays an important role in the sense that it creates a localized and extended high-intensity interaction region [19]. The spectrum at the output of the sample is collected with a lens and a fiberbased spectrometer. A filter with a flat response in the visible-near-UV region is placed before the spectrometer in order to reduce the input pump intensity without affecting the shape of the spectrum between 300-720 nm. The input pulse energy is varied from $10-50 \mu \mathrm{J}$, at which point the input pulse is in a strongly nonlinear regime and develops a complex and structured spectrum. Generation of negative RR modes is observed at intermediate energies $\sim 15 \mu \mathrm{J}$. Examples of the resulting spectra for varying input energies are shown in Fig. 2(a). The spectra are vertically displaced in order to render them visible. At lower energies $(12-14 \mu \mathrm{J})$ the output spectrum shows a distinct single peak that shifts to shorter wavelengths with increasing input energy. This process has been described in detail [20] in similar conditions and is a direct manifestation of the formation of a steep shock front on the trailing edge of the pump pulse. As energy is increased, the shock front steepens and the spectral peak shifts toward shorter wavelengths. Between 15 and $\sim 20 \mu \mathrm{J}$ input energy, a

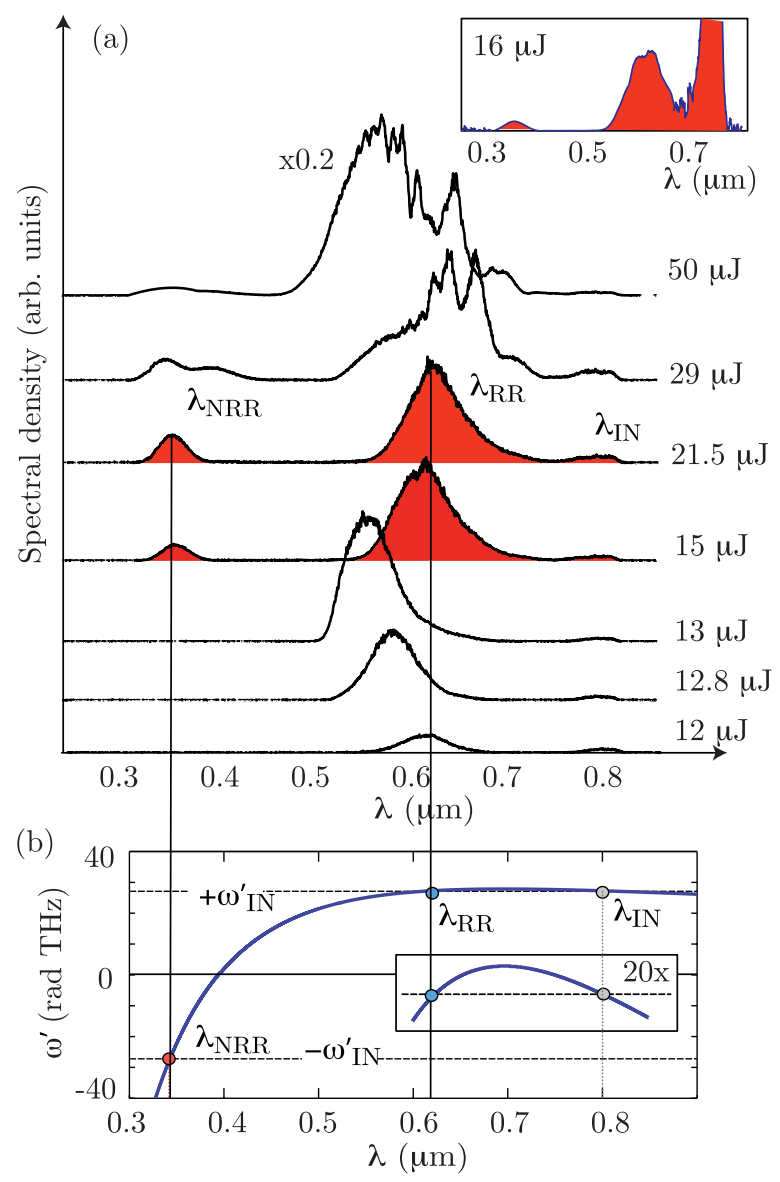

FIG. 2 (color online). Experimental results for negative RR generation in bulk $\mathrm{CaF}_{2}$. (a) Measured spectra for increasing input energies (indicated next to each curve). The spectra are vertically displaced to increase visibility. The inset shows a sample spectrum (16 $\mu \mathrm{J}$ input energy) corrected for the filter response. (b) $\mathrm{CaF}_{2}$ dispersion relation in the comoving frequency versus laboratory-frame wavelength coordinates, $\lambda=$ $2 \pi c /|\omega|$. Positions of the predicted RR and negative RR spectral peaks are indicated. The inset is a $20 \times$ enlargement of the curve around the $\lambda_{\mathrm{RR}}$ wavelength. 
different regime sets in, characterized by two distinct peaks in the spectrum that do not shift with increasing energy. The first peak is located around $620 \mathrm{~nm}$; the second is much weaker and is located around $341 \mathrm{~nm}$ wavelength. Examples of these spectra (15 and $16 \mu \mathrm{J})$ are shaded in red in the figure. At higher input energies, the pulse starts to develop complex dynamics, typical of the filamentation regime, during which the pulse breaks up and creates a broad-band, highly structured spectrum known as whitelight supercontinuum [21]. We focus our attention, for example, on the spectrum measured for an input energy of $16 \mu \mathrm{J}$ : the spectrum is not substantially modified if we account for the filter response, as shown in the inset to Fig. 2(a). Three clear peaks are indicated with $\lambda_{\mathrm{IN}}, \lambda_{\mathrm{RR}}$, and $\lambda_{\mathrm{NRR}}$, and we identify these with the IN, RR, and negative RR modes, respectively. Indeed, these peaks correspond exactly to the positions for the RR and negative RR modes given the IN mode and the dispersion relation for $\mathrm{CaF}_{2}$ [22], as shown in Fig. 2(b).

We note that attempts to generate similar features in other glasses or media (e.g., BK7, fused silica, and water) failed. Spectral broadening through self-phase-modulation and the steepness of Kerr-induced shock fronts are both strongly limited by dispersion. Our experiments in fused silica and water (data not shown) showed that even at the highest input energies, spectral broadening exhibited a sharp cutoff around $\sim 450 \mathrm{~nm}$, whereas the negative RR peak was, in all cases, predicted to appear at shorter wavelengths. On the other hand $\mathrm{CaF}_{2}$ (as other fluoride glasses) is quite unique, as it exhibits significantly lower dispersion [22], in particular in the UV spectral region, and thus allows the formation of steeper shock fronts and broader continua. In this specific case, it allows a relatively efficient excitation of the negative RR peak in the UV.

In a second experiment, we sent 7-fs light pulses, centred around $800 \mathrm{~nm}$, with a $77-\mathrm{MHz}$ repetition rate, into a fused silica photonic-crystal fiber (that has anomalous dispersion at $800 \mathrm{~nm}$ ). Photonic-crystal fibers have the advantage of enhanced nonlinear effects due to tight mode confinement, combined with a remarkable flexibility in tailoring the waveguide dispersion, that can therefore strongly modify the corresponding bulk medium dispersion and, thus, allow observation and control of a variety of novel effects. (See Ref. [5] for an extensive review.) We selected fibers where the spectrum of the incident light lies in a region of anomalous group-velocity dispersion such that it can propagate as a solitonlike pulse. The 7-fs input pulses are coupled into the fiber using a $90^{\circ}$ off-axis parabolic mirror. We estimate the coupling efficiency to be $20 \%$. In a preliminary experiment, we confirmed that most of the RR emerged using only a few millimeters of fiber. Therefore, short pieces of fiber of approximately 4-5 mm are used. Figure 3 shows the output spectrum after $5 \mathrm{~mm}$ of fiber for three different input energies $(246,324$, and $366 \mathrm{pJ})$. The ultraviolet part of the spectrum [Fig. 3(a)] was measured with a monochromator and a photomultiplier tube. It shows a clear peak that we identify

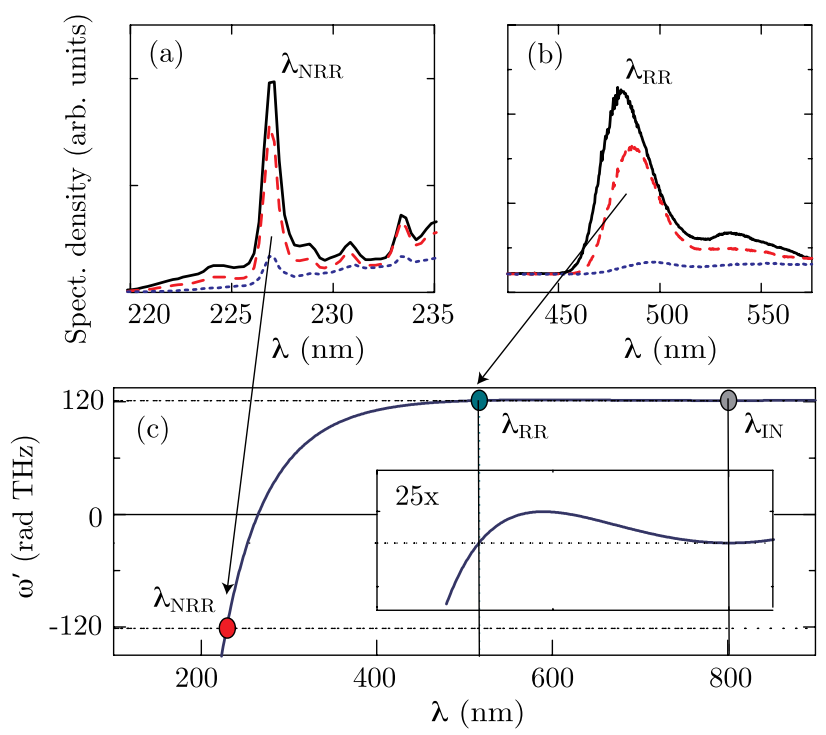

FIG. 3 (color online). Experimental results for negative RR generation in a photonic-crystal fiber. (a)-(b) Measured spectra in the visible and UV regions for three different input energies: $246 \mathrm{pJ}$ (dotted line), $324 \mathrm{pJ}$ (dashed line), and $366 \mathrm{pJ}$ (solid line). (c) Full fiber dispersion relation: positions of the predicted RR and negative RR spectral peaks are indicated. The inset is a $25 \times$ enlargement of the curve around the $\lambda_{\mathrm{RR}}$ wavelength.

with the negative RR mode. The part of the output spectrum that lies in the visible range, shown in Fig. 3(b), was measured with a compact CCD spectrometer. The peak observed here corresponds to the RR: the frequency of this mode shifts to shorter wavelengths with increased input pulse energy due to the nonlinear modification of the refractive index from the pulse [5]. Figure 1(c) shows the predicted RR and negative RR frequencies based on the dispersion relation for the photonic-crystal fiber. The measured peaks at $\lambda_{\mathrm{RR}}$ and $\lambda_{\mathrm{NRR}}$ are, similarly to the bulk measurements, the main spectral features in the whole spectrum and both correspond very precisely to the predictions. We note that the negative RR peak does not shift noticeably with input energy because the nonlinear refractive index change from the pulse is negligible compared to the dispersive index changes in the UV.

Experiments were repeated for a series of photoniccrystal fibers, as shown in Table I: NL-1.6 615 and NL1.5590 (used for the data shown in Fig. 3) consist of a solid silica core surrounded by a hexagonal pattern of air holes [23], and the fiber indicated with NL-1.5 670 consists of a

TABLE I. Predictions of $\lambda_{\mathrm{RR}}$ and predictions and measured values for $\lambda_{\mathrm{NRR}}$ for the three fibers used in the experiment.

\begin{tabular}{lccc}
\hline \hline Fiber & $\lambda_{\mathrm{RR}}$ & $\lambda_{\mathrm{NRR}}$ pred. & $\lambda_{\mathrm{NRR}}$ meas. \\
\hline NL-1.6 615a & $542 \mathrm{~nm}$ & $233.4 \mathrm{~nm}$ & $233.1 \mathrm{~nm}$ \\
NL-1.6 615b & $542 \mathrm{~nm}$ & $233.3 \mathrm{~nm}$ & $232.1 \mathrm{~nm}$ \\
NL-1.5 590 & $516 \mathrm{~nm}$ & $228.7 \mathrm{~nm}$ & $227.0 \mathrm{~nm}$ \\
NL-1.5 670a & $478 \mathrm{~nm}$ & $221.4 \mathrm{~nm}$ & $218.1 \mathrm{~nm}$ \\
NL-1.5 670b & $480 \mathrm{~nm}$ & $221.8 \mathrm{~nm}$ & $218.9 \mathrm{~nm}$ \\
\hline \hline
\end{tabular}


solid silica core surrounded by a cobweb of silica strands. The size and spacing of these holes and the thickness $(\sim 1 \mu \mathrm{m})$ of the strands determine the dispersion profile of the fiber. The letters $a$ and $b$ in the fiber name indicate measurements performed along one of the two orthogonal polarization axes of the fiber. The table lists the wavelength of the negative RR emission predicted from the respective dispersion relations compared to the actual measured wavelengths: as can be seen, very good agreement is obtained in a variety of settings.

In closing, we note that although four-wave mixing (FWM) could in principle occur between the RR and IN modes, leading to a further blueshifted peak (e.g., Ref. [4]), a simple calculation shows that in the fiber case this gives emission at $180 \mathrm{~nm}$ and in the bulk case to emission at $230 \mathrm{~nm}$. Both sets of measurements therefore cannot be explained by FWM, which in any case would be strongly suppressed by the severe wave-vector mismatch at these frequencies.

Conclusion.-Frequency conversion through a resonant transfer of energy from an input laser pulse to a typically blueshifted peak is a well-studied process in nonlinear optics and has attracted substantial attention in the last few years due to the high conversion efficiencies that are attainable with short pulses $[3,24]$ and, more recently, even to predicted mode-squeezing properties [25]. Here, we have shown how the same process generates a second, so-far-unnoticed peak that corresponds to resonant transfer of energy to the negative-frequency branch of the dispersion relation. The energy transfer is favored in the presence of steep shock fronts or, more generically, by a nonadiabatic variation within the pump pulse. Experiments were performed in both bulk media and waveguides with optimized dispersion landscapes so as to allow the process to occur with a relatively high efficiency. These results are not limited to nonlinear optics but are a general property of wave propagation in a dispersive medium: if the dispersion relation allows coupling between the positive and negative dispersion branches, then a similar excitation of negative modes may be expected. Relevant examples of experimentally realizable systems are gravity waves in water [26] or acoustic oscillations in Bose-Einstein condensates [27].

We thank Simon Horsely, Thomas Philbin, Scott Robertson, Philip Russell, and Sahar Sahebdivan for discussions. E. R. wishes to acknowledge Fondazione Cariplo, Univercomo, and Banca del Monte di Lombardia for financial support. Our work was supported by Heriot-Watt University, the University of St Andrews, and the Royal Society. Two of the authors (E. R. and J. M.) contributed equally to this work.

*d.faccio@hw.ac.uk

†fewk@st-andrews.ac.uk

[1] N. Akhmediev and M. Karlsson, Phys. Rev. A 51, 2602 (1995).
[2] P. K. A. Wai et al., Opt. Lett. 11, 464 (1986).

[3] L. Tartara, I. Cristiani and V. Degiorgio, Appl. Phys. B 77, 307 (2003).

[4] D. V. Skryabin and A. V. Yulin, Phys. Rev. E 72, 016619 (2005).

[5] J. Dudley, G. Genty, and S. Coen, Rev. Mod. Phys. 78, 1135 (2006).

[6] Localized Waves, edited by M. Zamboni-Rached, E. Recami, and H. E. Hernandez-Figueroa (WileyBlackwell, Chicester, 2008).

[7] M. Kolesik, E. M. Wright, and J. V. Moloney, Phys. Rev. Lett. 92, 253901 (2004).

[8] D. Faccio, M. A. Porras, A. Dubietis, F. Bragheri, A. Couairon, and P. Di Trapani, Phys. Rev. Lett. 96, 193901 (2006).

[9] D. Faccio, A. Averchi, A. Couairon, M. Kolesik, J. V. Moloney, A. Dubietis, G. Tamosauskas, P. Polesana, A. Piskarskas, and P. Di Trapani, Opt. Express 15, 13077 (2007).

[10] M. Kolesik, E. M. Wright, and J. V. Moloney, Opt. Express 13, 10729 (2005).

[11] M. Kolesik, D. Faccio, E. M. Wright, P. Di Trapani, and J. V. Moloney, Opt. Lett. 34, 286 (2009).

[12] M. Kolesik, L. Tartara, and J. V. Moloney, Phys. Rev. A 82, 045802 (2010).

[13] M. Born and E. Wolf, Principles of Optics (Cambridge University Press, Cambridge, England, 1999).

[14] L. Mandel and E. Wolf, Optical Coherence and Quantum Optics (Cambridge University Press, Cambridge, England, 1995).

[15] N.D. Birrell and P.C.W. Davies, Quantum Fields in Curved Space (Cambridge University Press, Cambridge, England, 1982).

[16] R. Brout, S. Massar, R. Parentani, and Ph. Spindel, Phys. Rep. 260, 329 (1995).

[17] D. V. Skryabin and A. V. Gorbach, Rev. Mod. Phys. 82, 1287 (2010).

[18] E. Rubino, F. Belgiorno, S. L. Cacciatori, M. Clerici, V. Gorini, G. Ortenzi, L. Rizzi, V. G. Sala, M. Kolesik, and D. Faccio, New J. Phys. 13, 085005 (2011).

[19] P. Polesana, D. Faccio, P. Di Trapani, A. Dubietis, A. Piskarskas, A. Couairon, and M. A. Porras, Opt. Express 13, 6160 (2005).

[20] D. Faccio, A. Averchi, A. Lotti, M. Kolesik, J. V. Moloney, A. Couairon, and P. Di Trapani, Phys. Rev. A 78, 033825 (2008).

[21] A. Couairon and A. Mysyrowicz, Phys. Rep. 441, 47 (2007).

[22] I. H. Malitson, Appl. Opt. 2, 1103 (1963).

[23] J. C. Knight, J. Arriaga, T. A. Birks, A. Ortigosa-Blanch, W. J. Wadsworth, and P. St. J. Russell, IEEE Photonics Technol. Lett. 12, 807 (2000).

[24] G. Chang, L.-J. Chen, and F. X. Kärtner, Opt. Express 19, 6635 (2011).

[25] T. X. Tran, K. N. Cassemiro, C. Soller, K. J. Blow, and F. Biancalana, Phys. Rev. A 84, 013824 (2011).

[26] S. Weinfurtner, E. W. Tedford, M. C. J. Penrice, W. G. Unruh, and G. A. Lawrence, Phys. Rev. Lett. 106, 021302 (2011).

[27] O. Lahav, A. Itah, A. Blumkin, C. Gordon, S. Rinott, A. Zayats, and J.Steinhauer, Phys. Rev. Lett. 105, 240401 (2010). 